\title{
nature
}

physics

\section{Here we go...}

\section{After almost three decades of preparation, CERN's Large Hadron Collider is turning on.}

The idea was there in 1977: to re-use a $27-\mathrm{km}$ circular tunnel on the Franco-Swiss border, built for a high-energy electronpositron collider, to house an even more powerful proton machine - the Large Hadron Collider ${ }^{1}$. By 1984 working groups were established to develop the proposal in earnest, and in December 1994 the project was ratified by the multinational council of CERN (the European Organisation for Nuclear Research). Now the LHC is about to come to life.

The first injection of proton beams into the collider is scheduled for later this month ${ }^{2}$; the first collisions to follow in the autumn. Thousands of physicists are on standby. The target beam energy is $7 \mathrm{TeV}$. The pressure inside the beampipe is $10^{-13}$ atmospheres. The collider's 9,300 magnets are supercooled to $1.9 \mathrm{~K}$. At full operation, the machine will produce up to $10^{9}$ collisions every second. Every statistic for this, the largest, most complex experiment ever undertaken, is extreme.

And in the extreme conditions created by the collision of $7-\mathrm{TeV}$ protons, the physics could be amazing. Proof of supersymmetry, of extra dimensions, evidence of particulate dark matter and for the origin of mass - all are real possibilities. It has been decades since a high-energy experiment has had such potential for discovery, and the chance to take a leap ahead of the theory.

But there are other possibilities to consider: microscopic black holes, strangelets, vacuum bubbles and magnetic monopoles. All of these could be formed in LHC collisions, and all could, in

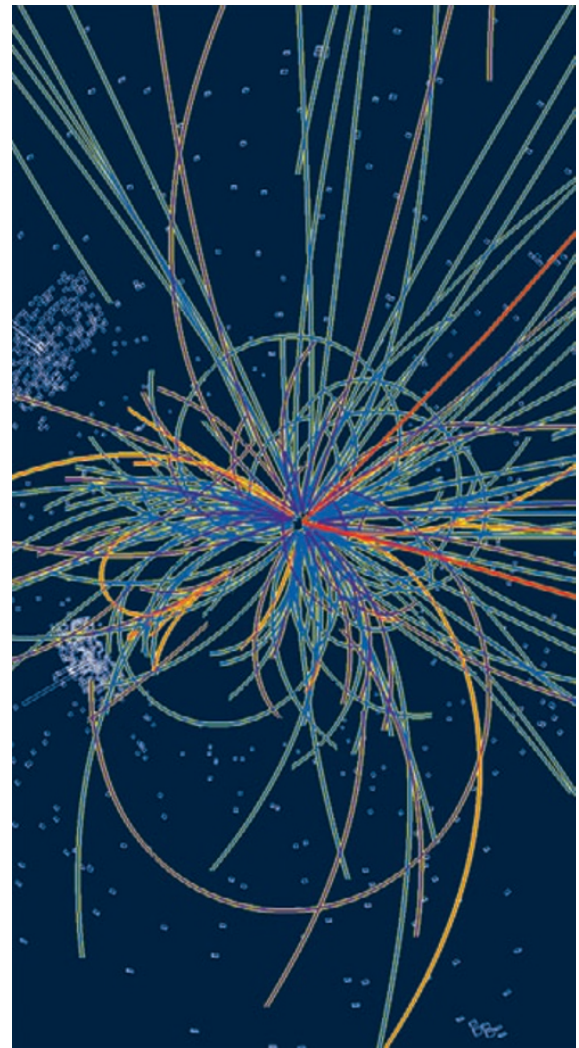

is that, on the evidence of the Universe around us (and particularly of cosmic rays that reach far higher energies than the LHC ever will), there is no tangible threat to our planet or our existence on it (at least, not from the LHC).

As the LHC goes into operation, it's time to stand back and fully appreciate what it represents. The LHC does not exist because there was any immediate, obvious spin-off to be had from the research, be it commercial or otherwise beneficial (although the example of history is that there will be rewards to come). The LHC exists because it could reveal knowledge to which we aspire. Aspiration doesn't necessarily come cheap - the price-tag for the LHC is somewhere in the region of $£ 5$ billion - but it's an investment to be proud of.

Robert R. Wilson, founding director of Fermilab, put it well. In 1969, appearing before the Congressional Joint Committee on Atomic Energy, he was questioned about the contribution of high-energy physics research to national security. "It has nothing to do directly with defending our country," said Wilson, "except to make it worth defending."

When the champagne corks fly at CERN - as they surely, and deservedly, will - the toast could well be raised to an international, inspirational project that is well worth defending.

Scare-mongering in some quarters, and an earlier tendency of CERN to champion the LHC as "recreating" the conditions after the Big Bang, has caused alarm.

CERN has, however, rightly countered such concerns by updating and publishing a thorough evaluation ${ }^{3}$ of the risks posed by the operation of the LHC. The fact

\section{References}

1. Nature Insight: The Large Hadron Collider. Nature 448, 269-312 (2007).

2. http://press.web.cern.ch/press/PressReleases/Releases2008/ PR05.08E.html

3. http://public.web.cern.ch/public/en/LHC/Safety-en.html 